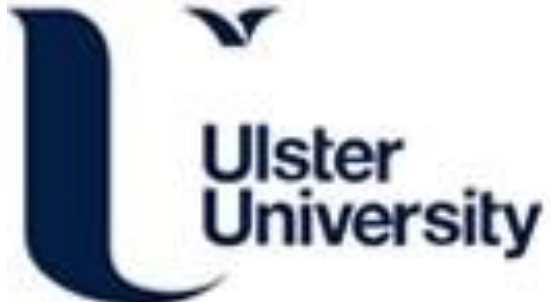

\section{Online Remote Control of a Wireless Home Automation Network}

Wade, J., Santos, J., \& Evans, N. (2009). Online Remote Control of a Wireless Home Automation Network. International Journal of Ambient Computing and Intelligence, 1(3), 39-52. http://www.igipub.com/articles/details. asp?id=35118

Link to publication record in Ulster University Research Portal

\section{Published in:}

International Journal of Ambient Computing and Intelligence

Publication Status:

Published (in print/issue): 01/01/2009

\section{Document Version}

Publisher's PDF, also known as Version of record

\section{General rights}

Copyright for the publications made accessible via Ulster University's Research Portal is retained by the author(s) and / or other copyright owners and it is a condition of accessing these publications that users recognise and abide by the legal requirements associated with these rights.

\section{Take down policy}

The Research Portal is Ulster University's institutional repository that provides access to Ulster's research outputs. Every effort has been made to ensure that content in the Research Portal does not infringe any person's rights, or applicable UK laws. If you discover content in the Research Portal that you believe breaches copyright or violates any law, please contact pure-support@ulster.ac.uk. 
nd Networking an MSc degree France. He is

of the Network. nd as direction :erland. She is orking, cuality c Networking conferences in vorks. She has ndation (ESF) Magazine, and r Networks of lat by Elsevier; us Computing ATC) both by ind Networks, cations Magaking and QOS EE PERCOM shop chair of WONS 2005 . hop and $A C M$ TCP of several as well as for Society, $A C M$ hoc networks.

\title{
Online Remote Control of a Wireless Home Automation Network
}

\author{
John Wade, University of Ulster; UK \\ Jose Santos, University of Ulster, UK \\ Noel Evans, University of Ulster, UK
}

\begin{abstract}
Embedded systems within home appliances are not usually mamufactured to operate in a networked environment; connecting supplementary hardware/software systems through a wireless, $P C$-controlled medium is necessary to enable full, efficient control of the ir functions from a remote location. Access to the home's central $P C$ may be gained via a local web server, giving Internet-based control from almost anvwhere in the world. The proposed system constitutes a significant improvement over those discussed in the literature to date, and reviewed here. It enables complex-appliance control in a secure and reliable portable-wireless enviromment. andwas developed using ASP.Net. The system was assessed for Received Signal Strength (RSS) in an environment more radio-hostile than that found in a typical household. The minimum RF level found at a transfer rate of $9.6 \mathrm{kbps}$ was $8 \mathrm{~dB}$ above the receiver's quoted sensitivity of $-103 \mathrm{dBm}$; this fadirng margin will increase in a normal household environment. [Article copies are available for purchase from InfoSci-on-Demand.com]
\end{abstract}

Keywords: Home Automation Network; Microcontrollers; Wireless Sensor Network

\section{INTRODUCTION}

As the cost of electronics components and microprocessors decrease, they are embedded increasingly into every day household items, e.g. microwave ovens, toasters, televisions and wáshing machines. Unfortunately it is still 
not possible to communicate with these appliances via a network, be that from within or outside the home. Although the appliances have embedded microprocessors to help control their onboard systems, they do not have the capability to communicate with a network of any kind. This is because there are no communication devices pre-installed and as yet no clear leader exists in home automation network protocols. Until this happens, true home automation networks cannot be fully realised. This. however, does not limit the ability to remotely control appliances in the home. To do this, domestic appliances can be grouped as:

1. Appliances that can only be turned on and off (Group 1).

2. Appliances that can be controlled in a more functional manner. such as televisions. DVD players and HiFi equipment (Group 2).

There are many reasons why the ability to remotely control the home is an advantage. In modern times. security and time management have become important issues. Houses may give an impression of occupation through controlled lighting and have their alarm. system monitored; kitchen tasks may be started remotely from a work location, saving time after the daily commute. A control network could also give the elderly and disabled a more independent way of life.

In section two, a background review of previous research into this topic is presented. Section three discusses the hardware design for the system investigated. Section four explores the software design for both the Internet interface and the firmware for the microprocessors. Section five discusses the testing protocol and results obtained from the system. Finally, section six presents conclusions and suggestions for future work.

\section{COMPUTER CONTROLLED HOME AUTOMATION}

There have been several approaches to computer controlled home automation: what follows is a review of the most significant and recent contributions.

Al-AliA.R.\&Al-Rousan M. (2004) developed a system built from a Java based user interface that was accessible through the World Wide Web. The system allowed the user to turn a cooker, light bulb or a fan on and off. This system used direct wiring from the computer to the appliances to exercise control over them: this approach is very costly due to the amount of cable needed to connect all the appliances found in a modern house. Also, appliances that fall into Group 2 could not be implemented in this system and introduction of new appliances into the household will result in further integration costs.

Sriskanthan N.. (2002), proposed a system that controlled home appliances from a PC using Bluetooth as the communications technology. As before, there was no consideration for

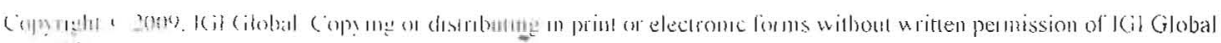
is probibital 
$\geq$ discusses

the system xplores the he Internet for the mi$\because$ discusses lts obtained section six uggestions

\section{.LED}

Jroaches to utomation: if the most ibutions. ר M. (2004) om a Java vas accesVide Web. sr to turn a on and off. $\mathrm{lg}$ from the to exercise rach is very ble needed - found in a zes that fall plemented ion of new I will result

proposed me appliuetooth as ology. As eration for those appliances that fall into Group 2 and could not be controlled over the Internet. By using Bluetooth there is also a limit to the number of appliances that can be controlled. According to Stallings (2002), up to eight devices can communicate in a small network called a piconet, and up to ten piconets can coexist in the same coverage range of a Bluetooth radio. This permits up to 80 appliances to be connected. Although a significant number at present, in the future this may become a limitation on the system. Another disadvantage of Bluetooth is that it allows ad-hoc networking. This enables an appliance that is Bluetooth enabled to establish an instant connection with another Bluetooth enabled appliance in the network when it comes into range. The main implication of this statement is that as new appliances are introduced into the home, the PC software has to be able to identify the appliance and either accept it or reject it automatically.

Bigio P. \& Cucos A. \& Corcoran P \& Chahil C \& Lusted K. (1999) designed and implemented a non-standard means of wireless networking suitable for home automation. Their system consisted of a low-power RF network broadcasting on $433 \mathrm{MHz}$, which again is license-free as it falls in the Industrial Scientific \& Medical (ISM) band according to the European Radio Commission Committee (2005).

The system is based on a master/ slave topology and is composed of a base station, relay units, and slave units. The base distinguishes between applica- tion requests and functions: application requests remain transparent to the radio system and therefore only the function of the appliance is broadcast. These broadcasts consist of frames/packets which have headers, body, trailer and a block/frame check for error checking. The relay units are used to extend the range of the base station, which has a range of $150 \mathrm{~m}$. This range is sufficient in most homes and therefore the relay units are not normally needed. The slave units are used to relay the information provided by the base station to their appliance. The network manager software controls all communications of the Radio Frequency (RF) network; it runs as a background service on the $\mathrm{PC}$ and is also responsible for granting or denying access to recently arrived slave units, depending on the unit being a legal part of the network. This system, whilst providing clear advantages over those previously proposed, still does not provide support for control over the Internet. However, it does show how useful and powerful using a nonstandard RF network for the communications medium can be, by allowing the designer full flexibility over the communications protocol.

Thomas R. (2000) designed a system that could decode signals from remote control units using the RC5 protocol, which is used by Philips, and the SIR C protocol used by Sony. This work highlights the problems that arise when trying to capture, decode and recreate remote control protocols. This system only dealt with two of the more 
common protocols: there are numerous others from a variety of manufacturers. Trying to produce a system that can recreate all protocols is not leasible. for two reasons. Firstly, from an economic point of view, a very wide range of remote controls from various manufacturers would need to be available so that signals may be captured and recreated. Secondly, the time needed to do this accurately is problematic; other workers dedicate much effort in this area [ref: Innotech Systems]. Therefore, it was considered more practical here to adopl a pre-designed universal remote control in the design.

Guan R \& Pruehsner WR \& Enderle JD $(2000)$ designed a system which used a GUI (Graphical User Interface) running on a PC to address the control problem presented by appliances that fall into Group 2. User input from a graphical representation of a remote control in the GUI sent data wirelessly to a PIC microcontroller via the serial port of the $P C$. This data was then transformed into special control commands fortuarded to a P P4001 universal remote control IC: this is preprogrammed to control most brands of appliances that have Infra Red (IR) remote controls. Although this approach negates the need to design a custom decoder, again there was no facility for control over the Internet and the PP4001 is expensive.

By extending the elements discussed above, it became possible to implement a system that is capable of controlling both groups of appliances over the Internet, thus providing a reliable and secure connection to all appliances within a wireless home network from almost anywhere in the world.

\section{HOME AUTOMATION NETWORK HARDWARE IMPLEMIENTATION}

The Home Automation Network is broken into 3 different sub-systems: the control unit (Base Unit), the Group 1 receiver units (GP1 Units), and the Group 2 receiver units (GP2 Units).

\section{Base Unit}

As shown in Figure 1, the Base unit is the main control center for the system; it communicates with the host PC (home

Figure 1. Base unit block diagram

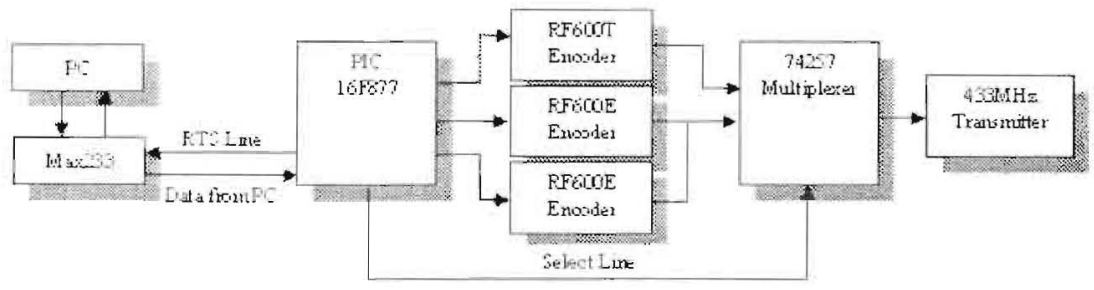

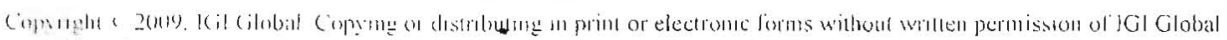
is poimbised 
controls. zgates the der, again ol over the xpensive. s discussed mplement ontrolling over the liable and ıppliances work from ld.

etwork is -systems: the Group i), and the : Units).

ase unit is zsystem; it PC (home
PC) via a serial port connection. The main computational element that controls communications is a PIC 16 F 877 microcontroller, which has a USART (Universal Synchronous Asynchronous Receiver Transmitter) built in. This receives data from the $\mathrm{PC}$ and is also responsible for RTS (Request to Send) handshaking between the Base unit and the PC. This ensures that no data is lost during communications due to buffer over-runs. All communications between the microcontroller and the PC use a MAX233 as an intermediate signal level converter.

Once data has been received by the microcontroller, it verifies that a full packet is present and the data is correct; data and packet structure will be discussed further in section 4. Once deemed correct, the microcontroller decides which group of appliances the data is intended for and processes it accordingly, either passing it to the Group 1 encoders or sending it through the USART to the Group 2 encoder, and on to the transmitter.

If the microcontroller decides that the data is intended for the Group 1 appliances, it firstly selects the appropriate input line of a digital multiplexer (Mux); this ensures that only data from the Group 1 encoders is sent to the transmitter. Then the data is output on ports $\mathrm{A}$ and $\mathrm{C}$ of the microcontroller, which are connected to the Group1 Manchester encoders (RF600E). These communicate with 4 decoders (RF600D), one on each GP1 unit. Matching the encoders to the decoders ensures that no other appliances outside of the system can be activated unintentionally and aids system security. The new encoded data is then sent via the Mux to the RTFQ1 (433 $\mathrm{MHz}$ FSK transmitter) [rfsolutions]; this has an estimated radiated power of $5 \mathrm{dBm}$ when using a $\lambda / 4$ antenna. The companion receiver has a sensitivity of $-103 \mathrm{dBm}$ for $9.6 \mathrm{kbs}$ operation. This implies that a maximum path loss of $108 \mathrm{db}$ may be tolerated.

If the microcontroller decides that the data is intended for the Group 2 appliances, then once again the correct input line to the Mux is chosen and the data is relayed via the USART to a serial encoder (RF600T). This is a serial version of the previous encoder which also uses Manchester encoding. The Encoder encapsulates the data into its own transmission packet, which consists of a 76 bit preamble, a 4-bit sync pattern, 1 start bit, 8 command bits, the data bits (size depends on the data sent from the microcontroller), and 8 checksum bits. This ensures that all data is received correctly by the decoder (RF600T) on the GP2 unit. This new encoded data is then sent to the FSK transmitter.

\section{GP1 Units}

The GP1 unit block diagram is shown in Figure 2. Each of the units is responsible for receiving control information from the Base unit and controlling the Group 1 appliances connected to them. Each GP1 unit can control 1 appliance.

When data is received by the FSK receiver the RF600D decodes it; if the

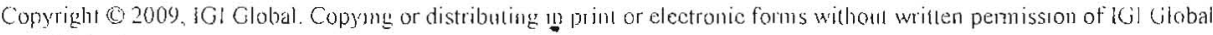
Is prolubiled. 
Fingure 2. (ipl unil block diagram

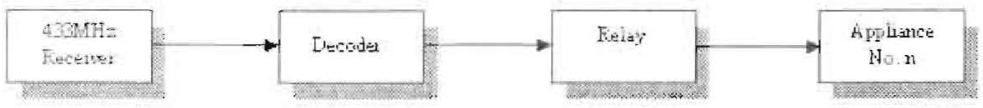

data is intended lor that unit then the decoder acrivates or deactivates an isolating relay. which in turn controls the retevant AC mains-connected appliance. The decoder has two modes of operation. momentary and latched: here the latter mode is used. Each time dala is received and verified for that specific decoder the output of the decoder is switched to the opposite state. i.e. if it was "on then it will be switched to "ofl". and vice-versa. This implies that the same data is transmitted for on and off. This element is part of the software design and will be discussed in section 4.

\section{GP2 Units}

Figure 3 shows a (jP2 unit, responsible for receiving control information from the Base and controlling the appropriate Group 2 appliance remote control. Each GP2 unit can control one remote.

When data is received by the FSK receiver the decoder (Rl:600T) ensures steering to that particular unit, and on to a PIC 16 F 877 microcontroller for further verification that it originated from the registered Base. If this is so, the PIC outputs the data on ports $B$ and D. These are connected to 4 separate 4016 quad bilateral switch banks. These switches are in turn hardwired to a Philips RU252 Universal Remote Control, which has the ability to control both televisions and VHS/DVD players/recorders from a wide number of manufacturers: up to 16 buttons on the remote may be controlled. In the prototype 15 were used for proof of concept. All 32 remote switches could be controlled with a few changes to the software and the addition of another 4 4016 devices.

\section{HOME AUTOMATION NETWORK SOFTWARE IMPLEMENTATION}

The software required to control the system was broken down into three different programs: to enablecommunications to the home server via a website, to control the microcontroller on the Base

Figure 3. (il'? amil block diugram

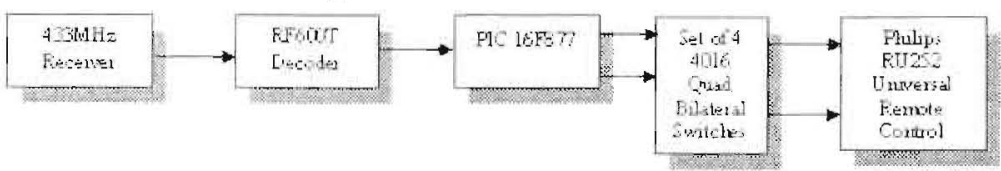

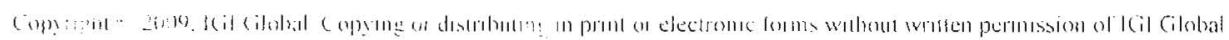
1) putumina 
unit, and to control the microcontroller on the GP2 units.

\section{Website Software}

sr for furated from s, the PIC D. These 016 quad switches ss RU252 which has levisions ersfroma s: up to 16 ontrolled. for proof hes could ges to the another 4

introl the three difamunicarebsite, to i the Base
The website used to access the system from a remote location is programmed in ASP.Net which forms part of the Visual Studio.Net package and the .Net framework. It allows the creation of Web applications and services that run under IIS (Internet Information services). IIS host Web applications on the Windows server, which in this case was based on the home PC. It manages the application, passes requests from clients to the application, and returns the applications responses to the client. ASP.Net is one of the most complete platforms for developing Web applications, making creation, debugging and deployment straightforward, as noted in Webb J. (2002).
Figure 4. System home page
When the user first accesses the website the Login screen is presented. Here the user is required to enter a user name and password; authorized user information is stored in a configuration file on the server. If the user is verified he / she is redirected to the home page, shown in Figure 4. When the Home page is loaded it accesses a database on the server that contains the current state of the Group 1 appliances on the system and displays this information under the Current State check boxes. If a check box is ticked then the appliance is "on" and vice-versa. The user can then update the state of the appliances by making adjustments to the New State check boxes. Once the user is satisfied with the changes the Update Appliances button is pressed; this sends the New State check boxes information back to the server, which then calculates two sets of data from this information.

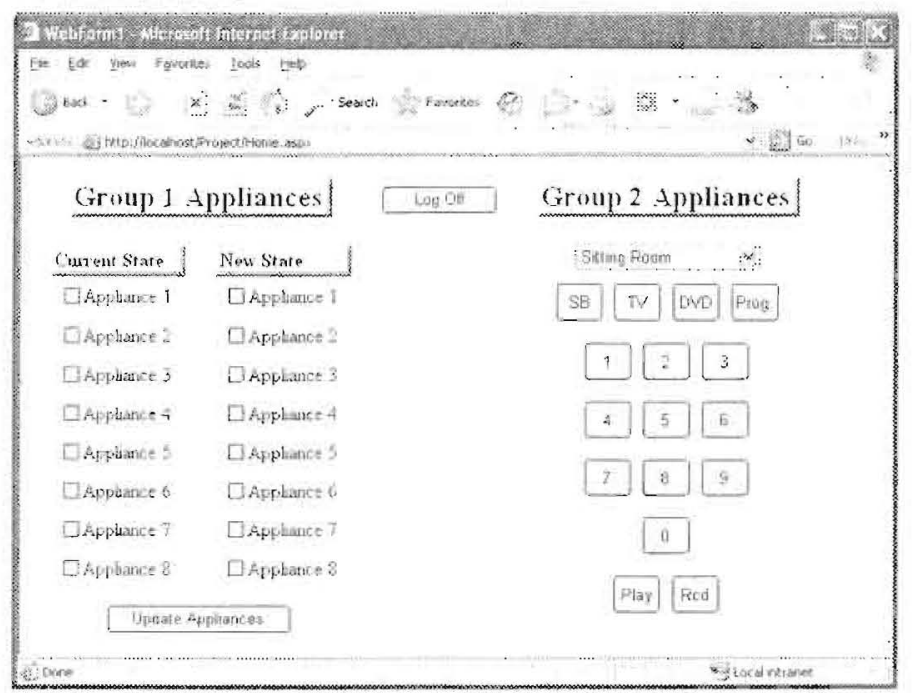

Copyight 2009 , IGI Global. Copying or distubuling an print or electronic fonns withoul writen permissron of IGI Global is prohibited. 
Firstly the New Stale information needs to beconverted into two numbers. each set ranging from () to 16 i.e. a 4-bit nibble. This is carried out as follows: appliances 1 to 4 represent the first set of data where appliance 1 is the least significant bit and appliance 4 is the most significant bit. [f an appliance is set to "on" then the corresponding bit is set, otherwise it is cleared. The same procedure is carried out for appliances 5 to 8. Once this set of data has been generated it is the necessary to produce another two numbers from the Current State check boxes. The two sets of data are then XOR"ed with one another and the result stored as Function bytes 0 and 1 . This ensures that if an appliance changes state then the corresponding bit for output will be set. allowing the Base unit to drive the encoders correctly, as discussed in section 3.1.

This data is then sent to the serial port where it is added 10 the transmission packet and transmitled to the base. This packet is made up of a start byte $=0$, address byte $=1$ and the two function bytes as shown in Table 1. The database is updated with the New State checkbox information and display in the Current Stalus check boxes on the retieshed page.

Tihle I. Transmisiven pucket firom server to herse mint

\begin{tabular}{|c|c|}
\hline Stut Eyto & 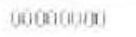 \\
\hline aditere Eytr & 00000001 \\
\hline Furntuor Byten & 0000 to 1111 \\
\hline Furtwo Eyte 1 & 0000 w11:1 \\
\hline
\end{tabular}

The user also has the option of controlling a Group 2 appliance via the onscreen remote control, by firstly selecting the area where the remote control is located from a dropdown list box. The address of the remote is defined by adding two to the index of the area selected in the list and is stored. When the user presses a button the address is sent to the serial port and forms part of the transmission packet. Each button has its own function defined for controlling the remote control and the two function bytes are exclusively defined within these functions. The Function 0 byte controls the first 8 switches and the Function 1 byte controls the next 8 switches. The screen is then refreshed. The transmission packet is similar to the transmission packet for the Group one appliances. Table 2 shows an example of the sitting-room remote control being accessed with the number 1 button pressed. Figure 5 shows the two function bytes and the corresponding switches that each bit controls.

If the Program button is pressed then buttons 1 and 3 are simultaneously accessed on the remote control; this allows the remote to enter program mode where a new device number may

Tuble 2. Transmission packet for GP2 Unit

\begin{tabular}{|c|c|}
\hline Sliart Eyte & 0อก000100 \\
\hline Adla es: Eytr & 001000010 \\
\hline Function Eyte 0 & 00010000 \\
\hline Furcton Egte 1 & 00บั0อบธ์ \\
\hline
\end{tabular}

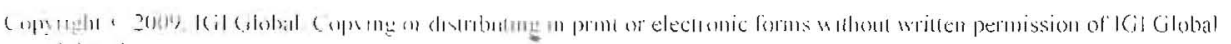
is proflubsed 
sption of iance via by firstly e remote 'down list is defined $f$ the area ed. When address is ns part of sh button I for conid the two $y$ defined unction 0 tches and the next 8 -efreshed. ailar to the jroup one I example ontrol ber 1 button o function switches

s pressed imultanee control; r progran mber may

Figure 5. Function bytes and their corresponding switches

\begin{tabular}{|c|c|c|c|c|c|c|c|c|}
\hline & Bit 0 & Bit ! & Eit.2 & Bit 3 & Bit. 4 & Bit 5 & Bit & Eit 7 \\
\hline Function Eyte 0 & SE & $T V$ & DVD & 0 & 1 & 2 & 3 & 4 \\
\hline Furiction Eyte 1 & 5 & 8 & 7 & 8 & 9 & Play & Record & Ne \\
\hline
\end{tabular}

be added and hence allow the remote to control a new appliance. On completion, 'Log off' permits a return to the Login screen.

\section{Base Unit Microcontroller Firmware}

When the microcontroller is started it clears the Request to Send (RTS) line leaving it ready for reception and waits until a byte has been received. The RTS line is then set, stopping all transmission from the PC. If this byte is $0 \times 00$ then it stores it as the Start byte, clears the RTS line and waits for the next byte, otherwise the byte is ignored, the RTS line cleared and the next byte checked for $0 \times 00$. The process continues with the next three bytes stored as Address, Function byte 0 , and Function byte 1 respectively. When the complete transmission packet has been received the program then checks which group of hardware has been addressed by subtracting 1 from the Address byte. If the result is 0 then Group 1 has been addressed, otherwise the system is addressing a Group 2 appliance.

If Group 1 has been addressed then Function byte 0 is passed to Port A which controls the first encoder, and the Mux line is cleared. A short delay of $0.5 \mathrm{~s}$ is called to allow the transmission of the signal, after which the port is cleared. Function byte 1 is then output on Port $\mathrm{C}$ and another delay of $0.5 \mathrm{~s}$ is called before the port is cleared.

If Group 2 has been addressed then the Mux line is set, the Start byte, Address byte, System Identification Number (SID) and the two function by tes are transmitted via the onboard USART to the serial encoder chip and then onto the RF transmitter. The SID number is preprogrammed into the microcontroller and consists of 4 bytes that provides up to $2^{32}$ different combinations. It is used as a unique system identifier for all the Group two remote controls. This ensures that only GP2 units registered with the same SID number as the base unit can be controlled by that base unit. thus preventing another system from accessing the GP2 units.

\section{GP2 Unit Microcontroller Firmware}

The start of the GP2 microcontroller program is similar to that in the Base unit, but with 4 extra bytes of data to be received before a complete transmission packet is collected: these are stored as SID 0 to 3. When the full packet has been received the microcontroller verifies that it has been addressed by subtracting the pre-programmed address from 
the address received. If the result is 0 its carries on. otherwise the packet is ignored and the microprocessor resets, ready for another transmission.

The next step is to verify that the SID number received matches the preprogrammed SID in memory: if so it continues. otherwise it again resets ready for another transmission. Once it has verified that the data is intended for the GP2 unit it checks if the program butcon was pressed on the website. This is done by subtracting the function 0 byte from $0 \times 50$. The result of pressing the program button is that Function byte $0=0 \times 50$. If the result is 0 then it enters the Program routine. Here Function byte $O$ is passed to Port $\mathrm{B}$ and a delay of $3 \mathrm{~s}$ is called before clearing the port. This activates buttons 1 and 3 on the remote control and makes it enter programming mode. If the result is not 0 then "Function 0" byte is passed to Port B for $0.5 \mathrm{~s}$. and the port cleared. This is followed with Function byte 1 being passed to port $D$ for $0.5 \mathrm{~s}$ before the port is cleared. Ports B and D are connected to quad bilateral switches that are hardwired to the remote control. In total. 253 GP2 units can be addressed by the Base unit.

\section{SYSTEM TESTING}

Hardware tests were carried out using a program written in VB6 that allowed the Start. Address. and Function byles to be explicitly entered as numbers before accessing the serial port. Transmission range in a hostile radio envonment was evaluated and software tests were carried out on the website.

\section{Transmission Characteristics Test}

Transmission range was tested by locating the transmitter and receiver units along different paths with a variety of obstacles, giving a significantly more radio-hostile environment than that found in a typical household, and measuring the Received Signal Strength (RSS) in each case. Figure 6 shows the location of the transmitter and receivers, along with the relevant obstacles in the path. The walls and columns are made of reinforced concrete with metal platting. The floor and ceilings are made of concrete. the overhang ceiling is made of minera! fiber tiles, the stud wall is made of a wooden frame covered in gypsum plaster board, and the elevator shaft wall is a red-brick construction.

As noted in Table 3, the received input level never fell below -94.2 $\mathrm{dBm}$. giving an $8.8 \mathrm{~dB}$ margin above the manufacturer's quoted sensitivity of $-103 \mathrm{dBm}$, for a transfer rate of 9.6 kbps. The fading margin in a normal domestic situation (with thinner concrete absorbers) is expected to be significantly greater.

\section{Hardware Test \& Software Tests}

To test the hardware, a prototype system was constructed. Three GP1 units were implemented using two encoders; the

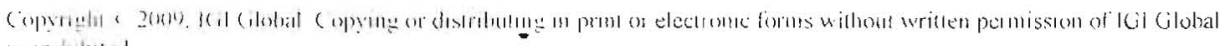
aprominted 
onment was

ts were car-

\section{eristics}

; tested by nd receiver with a varisignificantly nment than isehold, and mal Strength 6 shows the nd receivers, stacles in the uns are made h metal plat; are made of ng is made of wall is made $\mathrm{d}$ in gypsum tor shaft wall 1.

the received selow -94.2 largin above d sensitivity ar rate of 9.6 a normal doiner concrete : significantly

\section{ware Tests}

otype system P1 units were nncoders; the

Figure 6. Transmitter and receiver locations for RSS evaluations

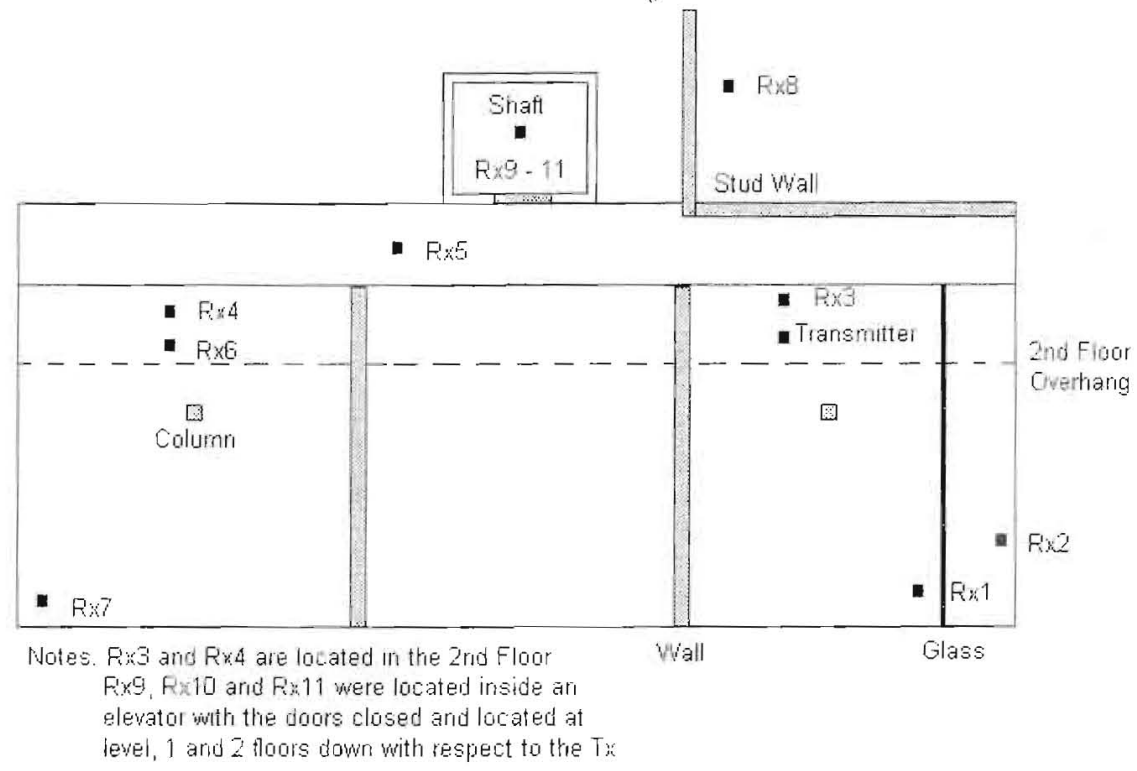

Table 3. Received signal strength measurements

\begin{tabular}{|c|c|c|c|}
\hline $\begin{array}{l}\mathrm{R} x \\
\text { No }\end{array}$ & $\begin{array}{l}\text { RSS } \\
(\mathrm{dBm})\end{array}$ & Propagation Path & $\begin{array}{l}\text { Direct Path } \\
\text { Length }\end{array}$ \\
\hline 1 & -57.52 & Around Square Column $\left(x \mathrm{~m}^{2}\right)$ & $9 \mathrm{~m}$ \\
\hline 2 & -52.7 .5 & Through Glass Panel (direcl Line of Sight) & $30 \mathrm{~m}$ \\
\hline 3 & -53.32 & Through Overhang Ceiling & $3 \mathrm{~m}$ \\
\hline 4 & -88.09 & Overhang Ceiling and 2 walts & $17 \mathrm{~m}$ \\
\hline 5 & -6.5 .15 & Through Wal! & $11.5 \mathrm{~m}$ \\
\hline 6 & -72.89 & Through 2 Walts & $19 \mathrm{~m}$ \\
\hline 7 & -85.75 & Through 2 Walls & $23 \mathrm{~m}$ \\
\hline 8 & -66.80 & Through 2 Siud Walls & $10 \mathrm{~m}$ \\
\hline 9 & -93.30 & Elevator Shaft & $13.5 \mathrm{~m}$ \\
\hline 10 & -93.38 & Elevator Shaft - I Floor Down & $14.2 \mathrm{nI}$ \\
\hline 11 & -94.20 & Elevator Shaft -2 Floors Down & $16.2 \mathrm{~m}$ \\
\hline
\end{tabular}

first encoder addressed 2 units, and the second addressed one unit. The intention was to prove that more than 1 decoder could be addressed by the same encoder and operated independently, and that the signal from the two encoders were separate, i.e.: if the first appliance was operated by the first encoder then the first appliance on the second encoder should not be operated at the same time as the signals should be different. The GP1 units were operated individually and then simultaneously by sending 
the appropriate commands from the test program.

During testing. the only problem that occurred was that if two sets of packets were sent one immediately after the other, one of the two encoders would lock up; this resulted in the base unit needing to be reset to correct the problem. However this will not represent a problem when the system is controlled through the website as it provides adequate time between packet transmissions due to page loading and refreshing.

The protolype also included a single (jP2 unit. Correct operation was confimed using a test program and the unit was also tested to verify that it would not respond to an address that was different to that stored in memory and that it would not respond to a packet with the incorrect SID number: This last test was achieved by replacing the microcontroller in the base unit with another one with a different SID number.

The web-based application program was first tested in a stand-al one mode for access security and forcorrect updating of the database with the "New States" when the "update" button was pressed on the application: then the prototype was fully connected to the host-PC and full control was achieved from the web-server:

\section{CONCLUSION}

The system developed is a very powerful home automation network that can be accessed from remote locations through the World Wide Web and operates in a wireless environment within the home. It consists of a website which is located on a home server and associated hardware, also connected to the server. The hardware is comprised of a Base unit that receives signals from the website and relays them on to the receiver units which are connected to the appliances. At present the system can control 8 appliances that can be turned on and off (GP1) and 253 appliances that are accessed via a remote control (GP2). Only minor software changes are needed to permit significant expansion. The system can operate in radio-hostile environments while maintaining a data rate of $9.6 \mathrm{kbps}$.

Although the system as described is not a fully-definitive home automation solution, it does serve as useful prototype. There are many improvements that could be made e.g.: the implementation of duplex communications between the Base and receiver units. The receivers could then inform the Base, and ultimately the user via the Web, that commands have been received and executed. This negates the need for the database as the receiver units could relay their status when the website is accessed. A related improvement is to grant users local control of the Group 1 appliances, with duplex links updating their Current State resulting from local switch changes within the home. The website program might also be enhanced to include a plan/layout of the home where each controlled appliance

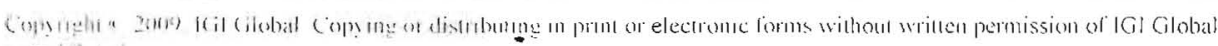
Nprotalisted 
locations and operənt within site which nd associted to the iprised of nals from on to the mected to iystem can be turned ippliances te control hanges are xpansion. lio-hostile ling a data zscribed is itomation ful protoments that mentation :tween the re receiv3ase, and Web, that :ived and need for nits could website is ment is to he Group uss updatting from the home. $t$ also be rout of the appliance is shown in its proper physical location: this decreases search time on user access by eliminating the need to search through check boxes and dropdown lists. Finally, the system developed can save time, help improve home security, and in general bring a better way of life to the end user.

\section{REFERENCES}

Al-Ali, A.R., \& Al-Rousan, M. (2004). Java-Based Home Automation System. IEEE Transactions on Consumer Electronics, 50(2), 498-504.

Bigioi, P., Cucos, A., Corcoran, P., Chahil, C., \& Lusted, K. (1999, August). Transparent, Dynamically Configurable RF Network Suitable For Home Automation Applications. IEEE Transuctions on Consumer Electronics, 45(3), 474 - 480.

European Radio Commissions Committee (2005) Frequencies and Standards. (online) http://www.ero.dk/doc98/official/ pdf/ REP083.PDF\#433050000. Accessed Sth July 2005.

Guan, R., Pruehsner, W.R., \& Enderle J.D. (2000. April). The Computerized Environmental Remote Control. Proceedings of the IEEE 26th Annual Northeast Bioengineering Conference (pp. 147-148).

RFSolutions. (2006) RTFQI Data Sheet. (on-line) http://www.rfsolutions.co.uk/ acatalog/DS069-7.pdf

Sriskanthan, N., \& Tan, K. (2002). Bluetooth Based Home Automation Systems. Journal of Microprocessors and Microsystems, 26, 281-289.

Stallings, W. (2002). Wireless Communications and Networking. New Jersey USA: Prentice-Hall.

Thomas, R. (2000, September) Remote Control IR Decoder: Everyday Practical Electronic's, 29(9), 698-701.

Webb, J. (2002). Developing Web Applications With Microsoft Visual Basic. Net and Microsoft Visual C\#. Net. Washington USA: Microsoft Press 


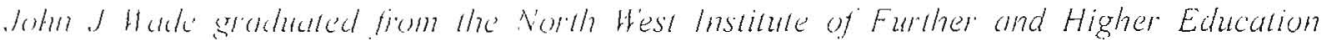
(A.HIF.H.E.) in 2001 with a BTec HND in electrical and electronic engineering. Wade is the holder of a firs class honours degree in electronics and compuning. with diploma in industrial

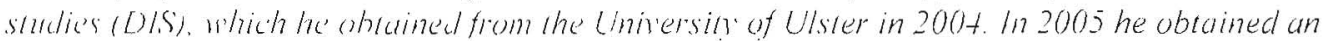
Alsc in computing and intelligent ststems fiom the University of Ulster: Wade is currently undergering a 1'hD in intelligent ststems at the University of Ulster 's Intelligent Systems Research ('nnte IISRC) where he is focusing on developing a biologically inspired araining algorithm for spiking newal newrorks. As well as newal nensorks bades main areas of incerest are, wireless commmnniculioms. and intelligenn sistems.

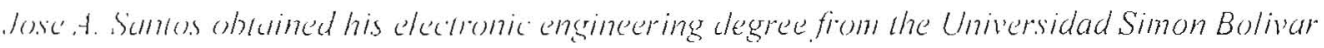

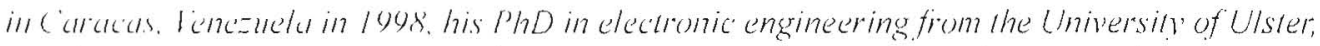
Northern freland. UK in 20102. and a PgCerl in higher education leaching from the University

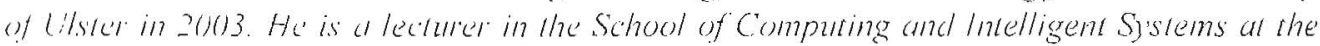

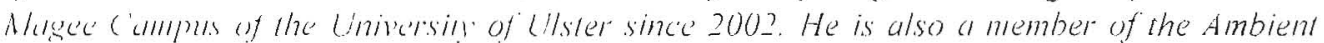

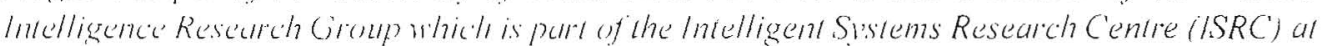
the lhiversing of Llster: His research interests lien in the area of umbient intelligence, RF and wircless sistems. sensor networks. hiotelemetry and biomedical sistems.

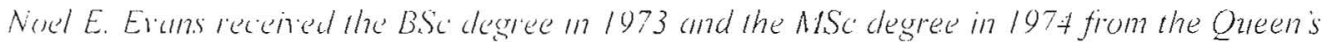

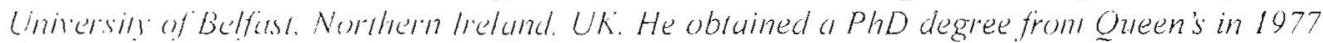
for work on programmathle trunswersul filters buill using intracell charge-coupled devices. Fol-

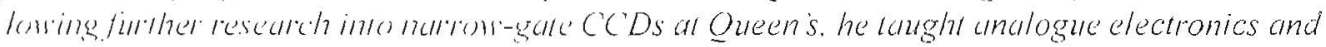

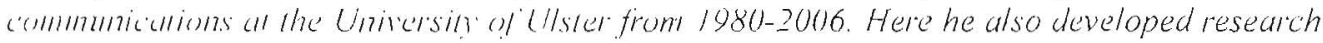
progerammess into hiomedical instramentation, human and animal phrsiological signa' acquisi-

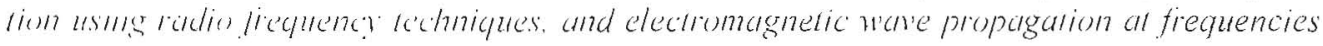
exkending aross the HF-l HF bands. He now works as an independent consultant in communiculions and celemerta:

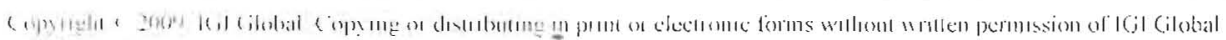
1. prituluticil 